\title{
Les Temps des territoires. Introduction du dossier thématique
}

Times of Territories. Introduction to the special section

Céline Clauzel, Jean Gardin, Catherine Carré, Anne Sourdril et Rhoda Fofack

\section{(2) OpenEdition}

Journals

\section{Édition électronique}

URL : http://journals.openedition.org/developpementdurable/12282

DOI : 10.4000/developpementdurable.12282

ISSN : 1772-9971

Éditeur

Association DD\&T

\section{Référence électronique}

Céline Clauzel, Jean Gardin, Catherine Carré, Anne Sourdril et Rhoda Fofack, « Les Temps des territoires. Introduction du dossier thématique », Développement durable et territoires [En ligne], Vol. 9, n '2 I Juin 2018, mis en ligne le 15 juin 2018, consulté le 20 avril 2019. URL : http://

journals.openedition.org/developpementdurable/12282 ; DOI : 10.4000/developpementdurable.12282

Ce document a été généré automatiquement le 20 avril 2019.

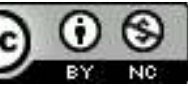

Développement Durable et Territoires est mis à disposition selon les termes de la licence Creative Commons Attribution - Pas d'Utilisation Commerciale 4.0 International. 


\section{Les Temps des territoires. Introduction du dossier thématique}

Times of Territories. Introduction to the special section

Céline Clauzel, Jean Gardin, Catherine Carré, Anne Sourdril et Rhoda Fofack

1 Le temps n'est pas conçu seulement de manière physique comme une dimension de l'existence. Il est aussi un construit social, divers (on songe aux régimes d'historicité de François Hartog, 2003) et contradictoire (on pense aux télescopages de temporalités longues et courtes sur les territoires qui en sont le substrat et le réceptacle). Le temps est objet de débats d'idées, voire de débats idéologiques, entre les partisans d'une accélération et ceux qui prônent une décélération (du changement, du progrès, de la modernité) ; autant de termes aussi intéressants et heuristiques que porteurs de pièges et de confusions, comme en témoignent les publications du manifeste accélérationniste ${ }^{1} \mathrm{ou}$, dans une mouvance inverse, de la revue Entropia ${ }^{2}$. Le temps peut alors être le prétexte à des injonctions (l'adaptabilité au changement par exemple) dont certains cherchent à renforcer l'acceptabilité, nous renvoyant non seulement à la dimension idéologique du temps, mais aussi à la question des instruments de sa mesure et de sa conception.

2 La question des modalités d'appréhension du temps dans la diversité des disciplines scientifiques revient régulièrement sur le devant de la scène, comme en atteste un grand nombre de rencontres et de publications échelonnées sur une vingtaine d'années ${ }^{3}$, tant en matière d'études de l'environnement que de politiques d'aménagement ou de santé : « Les temps de l'environnement» en 1997, «Villes et territoires réversibles» en 2010 ou le prochain congrès de l'Union géographique internationale, à Paris en 2022 qui aura pour thème "Le temps des géographes ». Cette question revient ainsi de manière récurrente dans les sciences sociales et les sciences de l'environnement, notamment en leur point d'intersection et, particulièrement, quand il s'agit de territoires et d'actions sur ces territoires.

3 Le présent dossier thématique de la revue Développement durable \& territoires souhaite ainsi montrer la diversité des approches en croisant les expériences et les recherches autour de la notion de temporalité. Il regroupe une sélection de textes issus d'un colloque qui s'est 
tenu les 9 et 10 mars 2017 à Nanterre, à l'initiative de l'UMR Ladyss'. À l'origine, l'objectif était d'interroger essentiellement la question des temporalités environnementales, dans leurs dimensions physiques et sociales. Dans un deuxième temps, nous avons souhaité ouvrir les communications aux diverses acceptions du terme temporalité. Il englobe une dimension prospective et rétrospective et invite au dialogue entre sciences de la nature, sciences sociales et expertise.

4 C'est bien la pluralité des approches et des champs scientifiques qui ont répondu présents. Ainsi, dans la sélection que nous présentons ici, des thèmes comme la patrimonialisation, les projets d'aménagement urbain, la construction de territoires marins sont abordés à côté de problématiques plus environnementales comme l'hydrologie de la Loire. Dans l'ensemble, les sciences de l'environnement n'ont représenté qu'une petite part des propositions. Cette diversité se retrouve dans les disciplines des auteurs qui sont pour moitié des géographes, le reste étant partagé entre aménageurs urbanistes, anthropologues, ingénieurs agronomes, sociologues, architectes, économistes et philosophes.

5 Si la question des temporalités a bien été au centre de toutes les contributions, celle du territoire n'a que rarement été posée sur le fond: le territoire va de soi (l'île). Parfois il renvoie implicitement à l'espace d'une communauté (ressource en eau); il peut aussi être un territoire de projet (d'aménagement), par les politiques publiques (les Plans de prévention du risque inondation - PPRI), ou simplement par les collectivités territoriales.

6 Les études de cas présentées dans le dossier opèrent des choix d'observation du temps, depuis les mesures des temporalités quotidiennes (les pas d'un humain, déjà étudiés par de Certeau, 1990) jusqu'aux métrologies utilisées dans les modèles numériques, destinées tant à améliorer les connaissances scientifiques qu'à gérer les interactions entre la nature et les sociétés.

7 Les questions d'évaluation sur lesquelles les chercheurs sont fréquemment sollicités renvoient inévitablement à la notion de temporalité : sur quel pas de temps évaluer les aménagements ou les politiques publiques? Comment prendre en compte, dans l'évaluation, l'évolution des comportements sociaux, des pratiques d'aménagement ou des technologies, notamment dans les outils de mesure?

Le dossier reprend les quatre thématiques du colloque :

- Les perceptions des changements environnementaux;

- Temporalité et aménagement des territoires : la question de l'état de référence dans les projets et leur réalisation;

- Articuler le temps et l'espace dans la compréhension des territoires: enjeux méthodologiques et outils ;

- Les politiques du temps.

9 C'est en suivant l'ordre de ces quatre thématiques, que les textes de ce dossier vont être successivement présentés.

\section{Les perceptions des changements environnementaux}

10 Les temporalités des changements environnementaux sont caractérisées par des emboîtements de cycles, de rythmes et d'échelles de temps; elles apparaissent comme multiples et discontinues. Depuis les années 1980, la classique question de l'opposition 
entre temps cyclique et temps linéaire est posée en des termes nouveaux : le changement climatique, par exemple, mobilise à la fois la question des cycles (e.g. du carbone) et celle de l'irréversibilité d'une évolution linéaire; les approches d'ingénierie écologique associées aux pratiques de conservation s'appuient, quant à elles, sur l'idée d'un fonctionnement systémique intégrant la notion de cycle ou de boucles. Le regard scientifique ou expert sur les questions de temporalité influe lui-même et produit des représentations sociales du changement environnemental et de ses rythmes. Les acteurs des territoires vont, pour leur part, opérer des diagnostics relatifs aux changements de leurs environnements ayant une temporalité propre. Toutes ces pratiques induisent-elles des représentations du temps singulières?

11 Dans les territoires, les changements globaux et les politiques ainsi que les actions pour un développement durable dessinent de nouvelles pratiques visibles dans les paysages urbains (gestion différenciée et valorisation de la biodiversité et du spontané) comme dans les paysages ruraux (culture biologique, agropastoralisme, sylvopastoralisme).

12 Nous posions plusieurs questions, dans le cadre de cette session du colloque, spécifiquement destinées à comprendre les perceptions locales des changements environnementaux et leurs impacts sur de potentielles stratégies d'adaptation : comment les habitants/acteurs des territoires perçoivent-ils ces changements et quelles relations entretiennent-ils avec ces variations des modes de valorisation des ressources? Les médias, les scientifiques, les gestionnaires et les entreprises relaient et formalisent un discours pour les citoyens sur les enjeux de durabilité des milieux. Les institutions insistent particulièrement sur les enjeux éducatifs. Comment les publics réagissent-ils à ces types de communication? Nous souhaitions ainsi observer la façon dont les conceptions du temps se construisent dans un dialogue horizontal autant que vertical, induisant des changements dans les taxinomies qui structurent les savoirs et les pratiques ainsi que des décalages dans les formes d'expertise, des conflits et des tensions.

13 Les deux articles en référence à cette thématique dans le dossier - celui de Camille Hochedez et Benoît Leroux (2018) et celui de Rhoda Fofack et al. (2018) - montrent qu'il existe à l'échelle locale une perception différenciée des temporalités et des risques associés aux changements et à leurs impacts sur les pratiques et les territoires. Camille Hochedez et Benoît Leroux (2018) précisent ainsi qu'en situation de catastrophe naturelle les changements environnementaux de type climatique ne sont pas toujours considérés par les acteurs locaux comme les causes des événements et des risques induits. Ces changements bénéficient d'une perception positive pour les habitants, dans le cas de cette étude, les viticulteurs. Les acteurs mentionnent les effets bénéfiques qu'ils auraient sur le temps du quotidien et à moyen terme, sur les pratiques viticoles ou les rendements des vignes. Ces articles montrent également comment, à l'échelle locale, changements environnementaux et changements sociaux sont considérés comme interdépendants et ne peuvent être abordés ou considérés de manière différenciée, notamment dans les politiques de gestion des territoires ou de conservation. L'article de Rhoda Fofack et al. (2018) saisit ainsi les liens et les contradictions entre perceptions de la disponibilité de l'eau au Maroc, pratiques traditionnelles, innovation technique et croissance agroéconomique.

14 Globalement les articles exposent une forte capacité d'action et d'adaptation des communautés locales aux changements qui affectent leurs territoires, tout en insistant sur une mise en concurrence des savoirs (locaux, scientifiques ou experts) et des conflits de légitimité entre les acteurs détenteurs des savoirs. Les auteurs évoquent aussi les 
perceptions contrastées, partagées par l'ensemble des acteurs (habitants, chercheurs ou experts), des effets des changements à court, moyen ou long terme, qui renvoient à la difficulté intrinsèque à se représenter les changements à des échelles de temps très longs.

\section{Temporalité et aménagement des territoires : la question de l'état de référence dans les projets et leur réalisation}

Les chercheurs et les gestionnaires insistent sur le fait qu'il n'existe pas d'état de référence absolu de l'environnement. Si cette affirmation est désormais partagée par l'ensemble des acteurs, pour autant la gestion environnementale et le gouvernement écologique des territoires demeurent conditionnés par l'utilisation de normes, de standards de qualité, d'outils d'évaluation de l'action publique, pilotés par un état de référence et des obligations de moyens et/ou de résultat pour l'atteindre. On renvoie pour exemple à l'atteinte d'un bon état des cours d'eau promue en 2000 par la Directivecadre sur l'eau, et qui s'impose pour 2018 à tous les États de l'Union européenne.

Les articles montrent différentes facettes de cet état de référence. L'article d'Alexandre Gaudin et Sara Fernandez (2018) présente le débit d'objectif d'étiage que l'agence de l'eau Adour-Garonne souhaite voir appliqué par l'ensemble des gestionnaires des cours d'eau le débit idéal d'une rivière en l'absence d'action humaine - et qui garantit le respect d'une vie aquatique. L'article de Julien Langumier et Margaux Knispel (2018) replace le rôle central de la crue historique de référence dans la gestion des risques: sa traduction cartographique dans les Plans de prévention des risques d'inondation communaux va définir des zones inconstructibles ou constructibles selon des prescriptions. Enfin, l'article d'Hervé Davodeau et de Régis Barraud (2018) retrace la genèse de deux états de référence qui aujourd'hui entrent en contradiction dans la gestion des paysages : celui d'une Loire sauvage porté par l'État et Voies navigables de France (VNF), et qui induit la suppression des épis du lit mineur du fleuve, et celui porté par des compagnies de navigation, des offices du tourisme, d'une Loire navigable et qui profite du chenal à épis.

Le territoire devient ainsi le moment et la condition d'une prise en charge de la nature, avec des modes d'action publique mêlant sciences et politiques. La traduction dans les politiques publiques des arguments scientifiques, des expertises permet de définir des principes et des outils d'action que les politiques publiques ont tendance à imposer, provoquant débats et contestations. Le débit d'objectif d'étiage renvoyant à une rivière idéale et ne pouvant être observé, l'agence de l'eau procède à un travail de reconstitution virtuelle par des modélisations hydro-écologiques des rivières, avec des modes discrétionnaires de ce qui entre ou non dans la modélisation, et naturalisant les choix passés de gestion et les négociations entre production d'hydroélectricité et irrigation agricole. Dans la gestion du risque inondation, le choix par les services de l'État d'un scénario de la catastrophe impose la référence d'un événement historique démesuré, qui s'écarte des expériences individuelles et des représentations collectives construites sur un événement vécu, et qui semble appliquer à l'excès un principe de précaution. Sur la Loire, l'approche techniciste de l'État et de VNF intervient au début de façon identique sur tous les épis de la Loire (par abaissement et raccourcissement), les auteurs soulignant toutefois un assouplissement récent des modes de faire de l'action publique, plus attentive aux usages et aux paysages. 

aménagements des sociétés pour envisager les conditions et les moyens d'une " gestion saine et durable » comme le proposait Jean-Paul Bravard (1998). Ici, les articles exposent la manière dont les acteurs articulent passé, présent et futur selon des chronologies particulières. La dimension temporelle s'incarne dans les discours d'acteurs à travers les accords (et les désaccords) pour donner du sens à la relation entre les collectifs et l'objet de nature visé par les politiques publiques (gestion de la sévérité des étiages, gestion du risque d'inondation, gestion paysagère et patrimoniale du fleuve). Ce récit doit être pris au sérieux par le chercheur « en dépit de la fiction qu'il construit dans la mesure où il légitime l'aménagement » (Davodeau et Barraud, 2018).

L'écologisation des politiques publiques place l'efficacité des actions pour la restauration d'un bon état des milieux naturels comme ultime juge de paix de l'action publique, et garante d'un mieux vivre ensemble. Hervé Davodeau et Régis Barraud (2018) évoquent " un contexte où les enjeux de biodiversité s'imposent à ceux du paysage ». Julien Langumier et Margaux Knispel (2018) précisent que les préconisations des PPRI sont applicables immédiatement : «Si la politique de prévention est justifiée par des objectifs de long terme, elle exerce un effet à court terme dès sa traduction dans une réglementation qui pèse sur les choix présents de développement des territoires. » Toutefois, Alexandre Gaudin et Sara Fernandez indiquent "qu'une telle solution ne peut cependant pas être mise en ceuvre aussi facilement que par le passé, et est aussi source de conflictualités ».

On peut alors se demander si, avec l'instauration d'un débit d'objectif d'étiage, la prise en compte du risque extrême, nous n'aurions pas affaire à un «nouveau futurisme » au sens où François Hartog (2003) l'a évoqué, parmi les catégories de rapport au temps des sociétés. Les trois articles font ressortir ce que les sociétés dans leur territoire décident d'engager ensemble dès maintenant pour transformer la portion d'espace terrestre qu'elles habitent et faire advenir un monde nouveau. Chaque article montre une facette différente, plus ou moins optimiste, de ce qui se joue aujourd'hui dans une éventuelle coconstruction des territoires.

21 Chaque article indique aussi un mode d'engagement des chercheurs :

- soit dans un travail critique des modes d'action publique. L'article de Alexandre Gaudin et Sara Fernandez démontre que "l'impératif scientifique qui entoure la définition des débits d'objectif d'étiage n'est pas tant porté par un souci de meilleure prise en charge de l'environnement que par un souci, de la part de l'administration déconcentrée de l'État et des conseils départementaux, de mettre en cohérence le temps court des crises et le temps long du compromis "; l'article de Julien Langumier et Margaux Knispel questionne pour sa part «la place centrale de l'État comme arbitre ou garde-fou du temps long ";

- soit pour Hervé Davodeau et Régis Barraud en proposant une médiation paysagère auprès des habitants, dans la mise en débat des trajectoires historiques des épis de la Loire, «questionnant les processus de patrimonialisation et de restauration écologique, notamment en réinterrogeant les états de référence sous-jacents aux représentations et à l'action publique». 


\section{Articuler le temps et l'espace dans la compréhension des territoires : enjeux méthodologiques et outils}

L'analyse des dynamiques spatio-temporelles soulève de nombreux enjeux méthodologiques et impose une réflexion sur les outils développés à ce jour. Ainsi les sources d'information et les instruments de mesure ou de suivi des faits sociaux et environnementaux (recensements, registres, enquêtes, techniques d'observation satellitaire, etc.) évoluent au cours du temps. Les nomenclatures s'appuient sur des catégories socialement et scientifiquement construites (urbain/rural, découpages spatiaux administratifs, catégories socioprofessionnelles, etc.) qui ne cessent de changer. Les biais méthodologiques liés à ces modifications sont présentés, sans que leurs conséquences soient suffisamment analysées.

Les auteurs interrogent précisément ces arbitrages méthodologiques, en lien avec la disponibilité des informations mobilisables: quelle granularité temporelle sélectionner pour restituer la trajectoire d'un territoire? Sur quelle base comparer des sources hétérogènes et/ou des typologies fluctuantes? Comment gérer l'incomplétude ou l'imprécision de la donnée? Dans quelle mesure les informations mobilisables permettent-elles d'appréhender les logiques spatiales et territoriales à l'œuvre? Comment pallier l'absence ou la rareté des données anciennes face à la profusion des données actuelles? L'ouverture de plus en plus grande à une masse et une variété d'informations numérisées ou le développement des comparaisons internationales incitent à revenir sur ces questions.

Amélie Robert et Sylvie Servain (2018) interrogent ces arbitrages à travers la reconstitution des dynamiques forestières sur le Domaine national de Chambord, un territoire circonscrit et dont l'originalité est d'être très documenté sur divers pas de temps. Les auteures questionnent le choix des temporalités à privilégier (celle des sylvosystèmes sur le temps long ou celles plus brèves des sociosystèmes), leur granularité, mais aussi la fiabilité des sources scripturales, iconographiques ou spatiales. Clémentine Périnaud (2018) explore ces mêmes questions en aménagement à travers la formalisation des transformations urbaines à une échelle séculaire. Les sources d'histoire urbaine relatives à l'action aménagiste (registres de séance du conseil municipal, dossiers d'instruction de projets, etc.) lui permettent ainsi de cartographier conjointement les dynamiques de recomposition de l'espace concret et de l'espace projeté.

Parallèlement à ces arbitrages se pose également la question de l'intégration du temps dans les analyses spatiales. Dans ce domaine, les outils de modélisation et de simulation dynamique permettent de tester des hypothèses, d'étudier les rétroactions, voire d'évaluer leur capacité de réversibilité. Les approches dynamiques offrent aussi l'opportunité de s'interroger sur la manière de représenter et visualiser les logiques spatio-temporelles des faits sociaux et environnementaux. Les outils de cartographie dynamique permettant de représenter des phénomènes de diffusion, des agrégats spatiotemporels ou de visualiser différents modèles de simulation se sont largement développés. constituer une aide à la décision publique? Laurie Tissière et ses co-auteurs (2018) 
proposent ainsi d'interroger les modalités d'intégration de l'espace et du temps dans la fabrication d'un récit qui s'opère dans le cadre d'un exercice participatif de géoprospective des pêches maritimes. Celle-ci est vue comme un guide pratique pour favoriser le débat et faire émerger des visions d'acteurs dans un contexte spatialisé et temporalisé. Sylvie Laroche (2018) appréhende la mutation des commerces à travers une approche historiographique de l'environnement urbain croisant données matérielles et immatérielles. Les temporalités sont restituées dans des transects urbains associant plans cadastraux, dessins et photographies in situ à plusieurs dates. Clémentine Périnaud mobilise pour sa part une cartographie 3D évolutive de villes, ou "SIG 4D », qui prend en compte le cycle de vie des objets et s'affranchit du découpage temporel induit par l'exploitation des sources planimétriques.

Dans toutes ces études, les outils sont mobilisés pour restituer les trajectoires passées des territoires, identifier des ruptures ou des tendances lourdes et proposer des scénarios d'évolution. Confrontés aux différents acteurs, voire co-construits avec eux, ces outils apportent des pistes stimulantes de réflexion pour l'aide à la décision.

\section{Les politiques du temps}

Les temporalités sont aussi des représentations fortement dépendantes des expériences historiquement situées de ceux qui les utilisent, y compris des scientifiques et des experts. Les temporalités ont donc à voir avec les régimes d'historicités de Hartog (2003), c'est-à-dire des manières situées d'appréhender les trois grandes catégories du passé, du présent et du futur. Les temporalités s'inscrivent dans tous les domaines de la vie publique ou privée, mais ne sont jamais abordées frontalement en des termes qui en font un sujet politique. Comment concevoir une chronopolitique qui permette de se réapproprier les débats sur les temps vécus? Lors du colloque, ce dernier atelier se proposait d'analyser les aspects normatifs, prescriptifs et descriptifs soulevés dans les autres ateliers, comme l'injonction au développement durable qui met au premier plan les questions de temporalités. La dette des générations présentes vis-à-vis des générations futures serait tout aussi bien une réponse qu'un des symptômes d'un certain état d'indécision contemporaine sur le temps.

Ceci oblige à un certain effort de réflexivité scientifique : les disciplines en lien avec les territoires se reconstruisent-elles avec le temps, en redéfinissant les temporalités et leurs pas de temps? Comment notamment certaines conceptions des temporalités percolentelles d'un domaine scientifique à l'autre en se rencontrant sur des objets communs? Nous songeons, à titre d'exemple, à la notion de climax et à ses tribulations : concept dominant (permettant de penser une nature stable, équilibrée, hors du temps et hors des influences anthropiques), puis concept mal aimé quand la nature pensée par les sciences sociales et les sciences de l'environnement s'est historicisée et socialisée ; et enfin concept persistant sous les questionnements liés aux états de référence et à une nature pristine.

Finalement, les trois textes rassemblés ici illustrent et éclairent ces questionnements, mais chacun de manière très différente. Véronique Zamant (2018) propose une lecture contextuelle de la patrimonialisation de Rio de Janeiro au titre du patrimoine mondial de l'humanité. Nous sommes dans ce texte confrontés à des acteurs de l'aménagement urbain et du marketing urbain qui semblent pris dans les ambiguïtés relevées par François Hartog: quelle forme de patrimonialisation peut convenir à une cité dont finalement bien peu d'éléments matériels sont jugés dignes d'une préservation? 
Comment tenir compte dans cette démarche patrimoniale des évolutions ou révolutions urbaines qui ne peuvent pas manquer d'intervenir dans un futur proche? La gestion prédictive (par la conception que les acteurs se font de l'avenir de la ville) vient télescoper l'image ou le modèle de la ville passée, à préserver des attaques du temps.

Pour Dominique Royoux (2018), c'est donc une politique du temps dont nous avons besoin. Dans son article sur l'articulation des temporalités de la vie quotidienne, il rappelle que l'époque contemporaine est marquée par un enchevêtrement des rythmes de la vie quotidienne qui se caractérise par une accélération des porosités entre temps du travail et temps hors du travail. Il s'attache alors à montrer comment se sont élaborées, depuis les années 2000, des réponses publiques aux questions d'articulation et de conciliation des temps sociaux de la part des collectivités territoriales, en France et en Europe. Tâche ardue, dans la mesure où les collectivités territoriales en France n'ont, selon lui, pas encore pris toute la mesure des conséquences des multiples désynchronisations à l'œuvre au sein des sociétés contemporaines des pays développés, laissant subsister de profondes inégalités entre individus et territoires. Il y a là matière à s'interroger. La seule réponse pour les collectivités territoriales est-elle de "prendre en compte » le dérèglement des temporalités collectives et s'y adapter?

Une autre réponse est proposée par Lisa Levy (2018) dans son article portant sur l'improvisation comme modèle pour l'action aménagiste. Elle distingue trois régimes de temporalité dans l'action aménagiste: la temporalité linéaire et prévisible de la planification ; la temporalité incertaine et itérative du projet ; la temporalité ouverte de l'improvisation. Elle soutient que la planification et le projet, tendus vers la maitrise des processus, ne peuvent accepter pleinement l'incertitude. À l'inverse, l'improvisation opère un renversement épistémologique et politique qui peut permettre de penser une ville ouverte. Il s'agit alors d'intégrer la surprise, l'ambiguïté, l'inachèvement, pour faire de l'indétermination un moteur de l'action.

Cette perspective nous rappelle les mondes liquides théorisés par Gilles Deleuze (1968), et pourrait en tant que telle être soumise à la même critique. Par exemple, le primat accordé au processus plus qu'au résultat ("la temporalité de l'improvisation se distingue par son absence de finalité »). Cela ne ressort-il pas d'une logique propre au Nouvel esprit du capitalisme décrit par Luc Boltanski et Ève Chiapello (1999) ? Ce primat ne résulte-t-il pas d'une récupération de la critique libertarienne vieille déjà de plusieurs décennies? L'auteure a conscience de cet écueil (« mis à part les managers nourris de néo-libéralisme qui la rangent parmi les best practices, voire en font un mot d'ordre, qui irait promouvoir l'improvisation?»). Il reste pour elle que l'improvisation est aussi « un geste de résistance, une force ouverte et attentive à l'autre, associée à des valeurs d'écoute, de partage, d'échange, de co-construction".

Pour conclure, l'appel fait aux chercheurs de passer des temps de l'environnement aux temps des territoires a bien été entendu. Les différents terrains étudiés ont rendu compte, chacun à leur manière, de l'intersection entre temporalités et territoires, à travers les espaces et leurs pratiques, les projets et les actions d'aménagement. L'ensemble des contributions a permis de couvrir les différentes pistes ouvertes dans l'appel à communication, aussi bien méthodologiques que réflexives. Elles contiennent à la fois des critiques des catégories et des outils utilisés pour penser les dynamiques spatio-temporelles des territoires, que des propositions pour continuer de réfléchir à ces dynamiques, en mobilisant les apports de l'analyse spatiale et la prise en compte d'indicateurs sociaux et environnementaux. Elles interrogent dans les territoires ce qui 
est mis ou non en débat des choix d'aménagement et le sens de l'action collective. Elles invitent enfin à reconsidérer la contribution des disciplines académiques et la place que peuvent prendre les chercheurs pour travailler collectivement le sens des actions que nous menons ensemble, quelque part sur la terre.

\section{BIBLIOGRAPHIE}

Beck C., Luginbühl Y., Muxart T. (dir.), 2006, Temps et espaces des crises de l'environnement, Versailles, Éditions Quæ, coll. « Indisciplines », 409 p.

Boltanski L., Chiapello È., 1999, Le nouvel esprit du capitalisme, Paris, Gallimard, 843 p.

Bravard J.-P., 1998, « Le temps et l'espace dans les systèmes fluviaux, deux dimensions spécifiques de l'approche géomorphologique », Annales de géographie, t. 107, n 599, p. 3-15.

Coste J., Fantini B., Lambrichs L. L. (dir.), 2016, Le concept de pathocénose de M. D. Grmek. Une conceptualisation novatrice de l'histoire des maladies, Paris, Droz, $346 \mathrm{p}$.

Davodeau H., Barraud R., 2018, « Quelle position adopter face au patrimoine qui vient ? La médiation paysagère pour mettre en débat les temporalités ", Développement durable \& territoires, vol. 9, n², http://journals.openedition.org/developpementdurable/12277.

De Certeau M., 1990. L'invention du quotidien, Paris, Gallimard, coll. « Folio essais », n 146, 416 p.

Deleuze G., 1968, Différence et répétition, Paris, PUF, 350 p.

Fofack R., Billaud J.-P., Kuper M., Petit O., 2018, « Analyse du basculement des modes d'extraction des eaux souterraines dans le Saïss (Maroc). Vers une reconfiguration des mondes des eaux cachées ? ", Développement durable \& territoires, vol. 9, n 2, http://journals.openedition.org/ developpementdurable/12197.

Frémont A., Allemand S., Heurgon E., Fixot A-M., Levêque J., 2008, Aménagement du territoire : changement de temps, changement d'espace, Caen, Presses Universitaires de Caen, $382 \mathrm{p}$.

Gaudin A., Fernandez S., 2018, « En attendant les barrages. Gouverner les temporalités de la gestion de la pénurie en eau dans le sud-ouest de la France », Développement durable \& territoires, vol. 9, n², http://journals.openedition.org/developpementdurable/12230.

Hartog F., 2003, Régimes d'historicité : présentisme et expériences du temps, Paris, Seuil, 272 p.

Hochedez C., Leroux B., 2018, “ “Après Xynthia... je ne suis pas inquiet, moi, la mer, ça ne me fait pas [peur]...”. Du déni à l'adaptation. Les viticulteurs de l'Ile de Ré face aux changements environnementaux ", Développement durable \& territoires, vol. 9, $\mathrm{n}^{\circ} 2$, https:// journals.openedition.org/developpementdurable/12392.

Langumier J., Knispel M., 2018, «L'anachronisme de la prévention des risques : les enjeux d'aménagement du présent réévalués depuis le futur des catastrophes possibles ", Développement durable \& territoires, vol. 9, $\mathrm{n}^{\circ}$ 2, http://journals.openedition.org/developpementdurable/12211.

Laroche S., 2018. «Représenter les temporalités : outils et méthodes pour saisir les phénomènes de vacance commerciale ", Développement durable \& territoires, vol. 9, n 2, http:// journals.openedition.org/developpementdurable/12153. 
Lefebvre H., 1992. Éléments de rythmanalyse. Introduction à la connaissance des rythmes, Paris, Syllepse, $116 \mathrm{p}$.

Levy L., 2018, « L'action sur les territoires face au défi d'une temporalité ouverte. L'improvisation comme modèle pour l'action aménagiste ? ", Développement durable \& territoires, vol. 9, $\mathrm{n}^{\circ} 2$, http://journals.openedition.org/developpementdurable/12236.

Périnaud C., 2018. Changement urbain et imaginaires aménageurs : une approche par l'exploration spatio-temporelle des sources d'histoire urbaine dans un SIG 4D », Développement durable \& territoires, vol. 9, $\mathrm{n}^{\circ}$ 2, http://journals.openedition.org/developpementdurable/12243.

Robert A., Servain S., 2018, « Retracer les temporalités d'une forêt emmurée. Le Domaine national de Chambord, les limites d'un exemple bien documenté " , Développement durable \& territoires, vol. 9, $\mathrm{n}^{\circ}$ 2, http://journals.openedition.org/developpementdurable/12163.

Royoux D., 2018, « L'articulation des temporalités de la vie quotidienne : nouveaux défis territoriaux des politiques publiques locales ", Développement durable \& territoires, vol. $9, \mathrm{n}^{\circ} 2$, URL : http://journals.openedition.org/developpementdurable/12265.

Tissière L., Michel C., Mahévas S., Trouillet B., 2018, « Raconter l'espace en jouant avec le temps. Exemple d'un exercice participatif de géoprospective des pêches maritimes ", Développement durable \& territoires, vol. $9, \mathrm{n}^{\circ}$ 2, http://journals.openedition.org/developpementdurable/12255.

Zamant V., 2018, « Mésentente temporelle. La fabrique du patrimoine culturel urbain de Rio de Janeiro à l'épreuve du temps des territoires des cariocas ", Développement durable \& territoires, vol. $9, \mathrm{n}^{\circ}$ 2, http://journals.openedition.org/developpementdurable/12173.

\section{NOTES}

1. http://www.multitudes.net/manifeste-accelerationniste.

2. http://www.entropia-la-revue.org/spip.php?article131.

3. On peut citer entre autres le colloque Les temps de l'environnement en 1997 ; les actes du colloque Temps et espaces des crises de l'environnement (Beck et al., 2006), ceux du colloque Aménagement du territoire: changement de temps, changement d'espace (Frémont et al., 2008), la journée des jeunes chercheurs de l'Institut de géographie Temps, rythmes et temporalités en géographie en 2011 ; la journée d'étude en sciences sociales Temporalités: regards croisés en sciences sociales en 2013, le colloque Géohistoire de l'environnement et des paysages en 2016, le colloque The history of pathocoenosis of the Mediterranean area : Diseases, environment, civilizations en 2010 (Coste et al., 2016).

4. http://www.ladyss.com/colloque-les-temps-des-territoires.

\section{RÉSUMÉS}

Ce dossier thématique rassemble une sélection des contributions au colloque «Les temps des territoires » organisé par le LADYSS en 2017. Il présente une pluralité d'approches centrées sur les temporalités des territoires, décrites à travers les espaces et leurs pratiques, les projets et les actions d'aménagement. Ces approches issues de différentes disciplines permettent de questionner les catégories et les outils conçus par les experts, pour mesurer le temps et analyser 
les dynamiques spatio-temporelles. Il s'agit plus particulièrement d'interroger l'usage récurrent d'un état de référence pour mener un projet, ou de discuter l'injonction à l'adaptation au changement climatique. Elles examinent le sens que les habitants et acteurs donnent alors aux trajectoires de leurs territoires et aux constructions - rétrospectives, prospectives - produites. Cet ensemble de textes a une ambition méthodologique et réflexive sur ce qui est mis en débat dans les choix d'aménagement et sur le sens donné à l'action collective, ainsi qu'à la place que peuvent y prendre les chercheurs.

This special section brings together a selection of contributions to the "Les temps des territoires" conference organized by the LADYSS laboratory in 2017. It presents a plurality of approaches centered on territories' temporalities, described through the spaces and their uses, projects and development actions. These approaches from different disciplines question the categories and the tools designed by experts, to measure the time and to analyze the spatio-temporal dynamics. It discusses notably the recurrent use of reference conditions to conduct a project, or the injunction to adapt to climate change. These papers examine the meaning that inhabitants and actors give to the territories trajectories and the resulting retrospective or prospective constructions. This set of proposals has a methodological and reflexive ambition: what is debated in development choices and the meaning given to collective action, as well as the place that researchers can take there.

\section{INDEX}

Mots-clés : temporalité, territoires, action, état de référence, prospective, dynamiques sociospatiales, trajectoires

Keywords : temporality, territories, action, reference condition, prospective, socio-spatial dynamics, trajectories

\section{AUTEURS}

\section{CÉLINE CLAUZEL}

Céline Clauzel est maître de conférences en géographie, université Paris-Diderot, UMR LADYSS, elle travaille sur la gestion de la biodiversité et des continuités écologiques. celine.clauzel@univparis-diderot.fr

\section{JEAN GARDIN}

Jean Gardin est maître de conférences en géographie, université Paris 1 Panthéon Sorbonne, UMR LADYSS, il travaille actuellement sur les espaces de la mise à mort des animaux et sur la circulation des normes en la matière. jean_gardin@yahoo.fr

\section{CATHERINE CARRÉ}

Catherine Carré est professeur en géographie, université Paris 1 Panthéon Sorbonne, UMR Ladyss. Elle travaille sur les politiques publiques dans le domaine de l'environnement, particulièrement sur l'eau et les milieux aquatiques, et les liens entre science et politique. catherine.carre@univ-paris1.fr 


\section{ANNE SOURDRIL}

Anne Sourdril est chargée de recherche au CNRS, UMR LADYSS, travaille sur les perceptions locales des changements environnementaux globaux au travers de l'observation de la biodiversité ordinaire. asourdril@gmail.com

RHODA FOFACK

Rhoda Fofack est docteure en sociologie de l'environnement de l'université Paris Nanterre et membre de l'UMR LADYSS. Ses recherches portent principalement sur les interfaces entre sociétés et ressources en eaux construites par le biais des objets et techniques. Elle travaille en outre sur les modes de gouvernance de ces ressources naturelles. fofack.rhoda@gmail.com 